Rev.MVZ Córdoba 17(1):2809-2811, 2012.

\title{
EDITORIAL
}

\section{Manuscrito duplicado o redundante: conducta impropia}

\section{Duplicate manuscript or redundant: misconduct}

La Organización de las Naciones Unidas para la Educación, la Ciencia y la Cultura (UNESCO, por su sigla en inglés), dentro de las categorías de artículos, los originales, los describe como aquellos en donde se informa sobre los resultados obtenidos, se describen métodos, aparatos, se expresan nuevas ideas, etc., y por lo tanto, se constituyen en la principal de las colaboraciones primarias destinadas a publicaciones periódicas. Un texto de un manuscrito pertenece a la categoría de "publicaciones originales" cuando éste contribuye a ampliar considerablemente el conocimiento o la comprensión de un problema y además, está escrito de tal manera que un investigador cualquiera pueda repetir los experimentos, cálculos o razonamientos teóricos del autor o autores y juzgar sus conclusiones, así como la precisión de su trabajo (1).

En Colombia, el Departamento Administrativo de Ciencia, Tecnología e Innovación (COLCIENCIAS) considera que el Artículo de investigación científica y tecnológica, es aquel que presenta de manera detallada los resultados originales de proyectos terminados de investigación. La estructura generalmente utilizada contiene cuatro apartes importantes: introducción, metodología, resultados y conclusiones, habida consideración de la revisión del estado del arte del tema plasmada en el cuerpo del manuscrito, así como en la sección de referencias (2).

Teniendo en cuenta las definiciones anteriores, se puede afirmar que el producto final de una investigación, debe ser en todos los casos, la divulgación de los resultados mediante la escritura y publicación de un artículo científico, el cual es clasificado o categorizado en el mundo académico, como artículo original. Esta clasificación, aunque parezca simple, significa entre otras muchas cosas, nada más y nada menos que los resultados son inéditos, fidedignos y reproducibles; es decir, que ellos no han sido publicados ni total ni parcialmente y que además, son ciertos, lo que responsabiliza de manera absoluta a los autores firmantes, pues en caso contrario, se incurriría en comportamientos aberrantes como el plagio o autoplagio, por referirnos a un solo calificativo de las conductas impropias relacionadas con la ética de las publicaciones.

Sancho (3) considera como publicación duplicada o repetida aquel manuscrito que es publicado varias veces y que en últimas se puede clasificar como un autoplagio. Igualmente comenta esta autora que quizás, desde el punto de vista de la ciencia, es el menor de los fraudes, ya que no distorsiona ni falsea los resultados obtenidos en el primer trabajo. No obstante, consideramos que desde la óptica de editores y usuarios de la información como son los lectores, esta práctica debe ser proscrita y rechazada tajantemente dentro de las políticas editoriales de todas las revistas científicas del mundo, sancionando de alguna manera a quienes incurran en ella.

De acuerdo con los Requisitos de Uniformidad de las Revistas Biomédicas -a los cuales se acoge la Rev.MVZ Córdoba- contenidos en las Normas de Vancouver, la publicación 
redundante o duplicada consiste en la publicación de un artículo que coincide sustancialmente con otro ya publicado. Adicionalmente, se enfatiza en dichas normas que los lectores de las revistas biomédicas -creemos que cualquiera- deben tener la garantía de que aquello que están leyendo es original (4). Por otra parte, el International Committee of Medical Journal Editors (ICMJE), clasifica como publicación duplicada o redundante aquella que se solapa o coincide sustancialmente con un manuscrito ya publicado, bien sea en versión impresa o electrónica, y que hoy día es relativamente fácil detectar esta última en la WEB, descubriendo por lo tanto a los infractores (5).

Los editores de seis Revistas de Cirugía Cardiotorácica (6), ante la gravedad y presencia indiscutible del mal, han discutido el tema y publicado las siguientes condiciones para declarar una publicación como redundante o duplicada: Hipótesis similar, la población de estudio o el tamaño de la muestra es similar, la metodología es idéntica o casi igual, los resultados son parecidos, como mínimo uno de los autores es común en ambas (o varias) publicaciones y por último, la novedad de la información proporcionada es mínima o casi nula.

Creemos que pueden existir muchas razones para desarrollar esta conducta impropia. Probablemente, este comportamiento en algunas casos se deba a diversas presiones a que son sometidos los investigadores por las mismas instituciones, entes financiadores, competencia entre investigadores o entre grupos de investigación, productividad científica, acceso a recursos, mejora del currículum vítae, clasificación interna de investigadores dentro de institutos, centros de investigación o universidades, así como mejoras laborales o ascensos en la jerarquía del organigrama institucional y laboral. Tampoco debemos desconocer que quizás en algunos casos pueda primar el interés económico sobre el académico y por lo tanto, algunos investigadores se ven tentados a incurrir en faltas a la ética de publicaciones, fraccionando sus investigaciones o como se comenta en los corrillos, optan por publicar manuscritos en serie, recurriendo al uso de sinónimos en apartes de los títulos o a pequeñas modificaciones en alguna sección o secciones del artículo, bien sea agregando o quitando pero conservando lo medular del primer manuscrito publicado.

Otra causa probable que también induce a los investigadores a realizar publicaciones duplicadas, es el desconocimiento de las normas que rigen las actividades de publicación y en especial en lo que tiene que ver con la ética, tal como lo consagra en sus directrices la World Association of Medical Editors (7) instando a los miembros de Comités Editoriales y a Editores de las revistas de dicha asociación -a la que también pertenece la Rev.MVZ Córdoba- para que desarrollen e implementen unas claras políticas editoriales, incluidas las faltas a la ética de la publicación en todos sus espectros, así como la sanción social o académica a que se hacen acreedores aquellos que caigan en esas conductas impropias.

También es evidente que existen áreas del la ciencia que pueden ser más vulnerables, en donde entran a jugar un papel importante la política, la comercialización, los medicamentos y la salud entre otras, en donde se pueden ver involucradas investigaciones clínicas con pacientes de diverso orden. En este sentido Sancho (3) comenta que puede existir presión para que se dupliquen los artículos con resultados positivos acerca de nuevos medicamentos o por el contrario, retrasar la publicación de los resultados negativos, sobreestimando así la eficacia y seguridad de los mismos, lo cual puede dar lugar a posteriores análisis incorrectos de riesgos-beneficios.

Es importante mencionar también, que bajo ciertas circunstancias es posible realizar publicaciones duplicadas, siempre y cuando las partes (autores y revistas) estén absolutamente de acuerdo. Por comentar un solo ejemplo de los múltiples que hay, un editor de una revista técnica, puede solicitar permiso para publicar un manuscrito ya publicado, al editor de una revista científica. Obviamente, la redacción del manuscrito para 
las condiciones de la revista técnica deberá ser modificada, teniendo en cuenta el público para el cual está dirigida. En cualquier caso, creemos que estos permisos deberán estar plenamente sustentados, oficializados y firmados por los interesados.

Con relación al oficio de editor, estas malas prácticas hacen perder dinero, tiempo y recursos de toda índole durante el proceso de sometimiento de un manuscrito, que dicho sea de paso; siempre es largo y desgastante para las partes. Además, también involucra a los pares, quienes realizan dicha labor solo por convicción, formación y colaboración académica, pues cuando mucho, reciben una mención de agradecimientos al final de cada número de la revista, habiendo invertido un valioso tiempo y esfuerzo que francamente agradecemos y valoramos, pero que para los infractores, no tiene valor alguno. Realmente, es una falta de respeto y consideración proponer para posible publicación un manuscrito duplicado o "seriado" como hemos querido llamarlo nosotros, pues con esta denominación, incluimos aquellos que superan la barrera de la duplicación.

En un país que parece haberse acostumbrado en la última década a ver actos de corrupción y faltas a la ética, la academia no puede seguir ese rumbo equivocado de ausencia de valores, de vida fácil y de menor esfuerzo para alcanzar lo que se quiere.

Queremos finalizar este editorial, evocando uno de los tantos proverbios populares: no basta parecerlo, hay que serlo.

Marco González T. M.Sc. Salim Mattar V. Ph.D.

\section{REFERENCIAS}

1. UNESCO. Guía para la redacción de artículos científicos destinados a la publicación. 2 ed. París: UNESCO, 1983. URL Disponible en: http://unesdoc.unesco. org/images/0005/000557/055778SB.pdf

2. Documento Guía. Servicio Permanente de Indexación de Revistas de Ciencia, Tecnología e Innovación Colombianas Base Bibliográfica Nacional BBN. Índice Bibliográfico Nacional Publindex - IBN. Bogotá: Colciencias, Publindex. URL Disponible en: http://201.234.78.173:8084/publindex/ docs/informacionCompleta.pdf

3. Sancho R. Publicación duplicada: [En línea]. Instituto de Estudios documentales sobre Ciencia y Tecnología - IEDCYT-CSIC. 2008 URL disponible en: http://www. Madrimasd.org/informacionIdi/análisis/ análisis/análisis. asp?id=36354

4. Estilo de Vancouver. Requisitos de Uniformidad para Manuscritos enviados a Revistas Biomédicas. URL Disponible en: http://www. fisterra.com/herramientas/recursos/ vancouver/\#CUESTIONESPREVIAS
5. International Committeee of Medical Journals Editors. Uniform requirements for manuscripts submitted to biomedical journals: Writing and editing for biomedical publication [documento en Internet]. Vancouver: ICMJE; 2008 [consultado: 5-04-2012]. URL Disponible en: http:// www.metodo.uab.cat/docs/Requisitos_ de_Uniformidad.pdf

6. Cho BK, Rosenfeldt F, Turina MI, Karp $R B$, Ferguson $T B$, Bodnar $E$, et al. Joint statement on redundant (duplicate) publication by editors of the undersigned cardiothoracic journals. Ann Thorac Surg $2000 ; 69: 663$.

7. World Association of Medical Editors. World Association of Medical Editors (WAME) recommendations on publication ethics policies for medical journals. Arch Med Res 2004; 35(4):361-67. 